\title{
Automatic target detection and localization using ultra-wideband radar
}

\author{
Dounia Daghouj ${ }^{1}$, Maroua abdellaoui ${ }^{1}$, Mohammed Fattah ${ }^{2}$, Said Mazer $^{1}$, Youness Balboul ${ }^{1}$, \\ Moulhime El Bekkali ${ }^{1}$ \\ ${ }^{1}$ National School of Applied Sciences, Sidi Mohamed Ben Abdellah University, Fez, Morocco \\ ${ }^{2}$ Superior School of Technology, Moulay Ismail University, Meknes, Morocco
}

\begin{tabular}{l} 
Article Info \\
\hline Article history: \\
Received Apr 29, 2021 \\
Revised Sep 18, 2021 \\
Accepted Oct 25, 2021
\end{tabular}

\section{Keywoords:}

Correlation peak

Detection

Localization

Target

Ultra-wide band radar

\begin{abstract}
The pulse ultra-wide band (UWB) radar consists of switching of energy of very short duration in an ultra-broadband emission chain, and the UWB signal emitted is an ultrashort pulse, of the order of nanoseconds, without a carrier. These systems can indicate the presence and distances of a distant object, call a target, and determine its size, shape, speed, and trajectory. In this paper, we present a UWB radar system allowing the detection of the presence of a target and its localization in a road environment based on the principle of correlation of the reflected signal with the reference and the determination of its correlation peak.
\end{abstract}

This is an open access article under the CC BY-SA license.

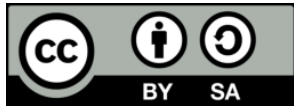

\section{Corresponding Author:}

Dounia Daghouj

National School of Applied Sciences, Sidi Mohamed Ben Abdellah University

Fez, Morocco

Email: dounia.daghouj@usmba.ac.ma

\section{INTRODUCTION}

Road safety is an important consideration in everyday life. Most vehicles are equipped with collision avoidance sensors such as laser scanners, cameras, and infrared sensors. These systems are used to detect obstacles, their positions, and speeds. They work reliably under certain conditions but suffer from several limitations in others. Factors such as open areas, fog, smoke, dust, dirt, lighting conditions, and reflections can lead to faulty sensor values. In addition, visual and infrared sensors must be classified close to the target, while radar sensors depend on the frequency of operation and can operate up to several hundred meters.

The term radar, translated into radio detection and ranging [1], [2], means detecting and estimating distance by waves; it is a device emitting and receiving electromagnetic waves, used to detect and locate objects in any space. The capacity of radar is determined according to the technology used, and as it is known, the most exploited technology in the current research work aimed at road safety is the ultra-wide band (UWB) [3], [4] since it allows detection [5] and localization with great precision and in any environment [6].

The detection of the various obstacles by an UWB system is based on the transmission and reception of brief duration pulses, of the order of a few Pico or nanoseconds [7], which requires a specific signal processing at a reception to properly exploit the reflected signals [8] and extract the maximum amount of information. In order to develop a UWB radar [9] system capable of detecting targets in urban areas, one of the main difficulties is related to the complexity of the propagation environment induced by the obstacles present in the scene, and generally, the target is not in the line of sight of the radar, which complicates the task more and more. The recent literature includes several works dealing with this problem [10], [11] and in this 
work, we explore a new solution based on an algorithm for the detection and localization of non-line-of-sight (NLOS) targets in urban areas based on the exploitation of signals propagating in a vehicle to vehicle channel, which follows a Rayleigh distribution and reflects the characteristics of the urban environment [12] and in particular the multipath phenomenon. This algorithm is essentially based on a simple and efficient processing using the correlation principle that allows testing the similarity between the reflected signals and the template to detect existing targets in the radar area with high accuracy and low cost.

This article focuses on the presentation of an algorithm, based on the determination of the maximum correlation peak, to determine the presence of an obstacle in the backscattered electromagnetic field while correlating the received signal with the reference and the localization of these targets detected by calculating the distance separating the radar from them. In the first part of this paper, we propose an algorithm that allows the detection of a target and its localization in a road environment based on the correlation technique, then we present the detection and localization method used by our UWB radar and at the end, we discuss the results of the simulation.

\section{THE PROPOSED ALGORITHM FOR DETECTING AND LOCATING TARGETS}

Obstacle detection and localization is a subject that interests many researchers and has been the subject of much work. Several algorithms have been proposed for the detection and localization of obstacles in the road domain and each algorithm has its strengths and weaknesses [13]-[15]. Most of these methods have somewhat complex architectures and require the fusion of radar with another type of sensor. In this work, the proposed algorithm shows a simple and efficient approach to signal processing; based on the coherent reception of the signal and the correlation of the received signal with the reference. Although the proposed algorithm contains a coherent receiver which is recognized by its power consumption and somewhat complex architecture, it can help to improve the signal-to-noise ratio (SNR) by the coherent integration of the signal and have a better sensitivity to maximize the probability of detection. The efficiency of this signal processing approach is verified by the detection simulations carried out. The presented algorithm is therefore capable of detecting and locating road obstacles and presents a solid basis for a future development of the field of classification. Figure 1 describes our target detection and localization algorithm for a UWB system.

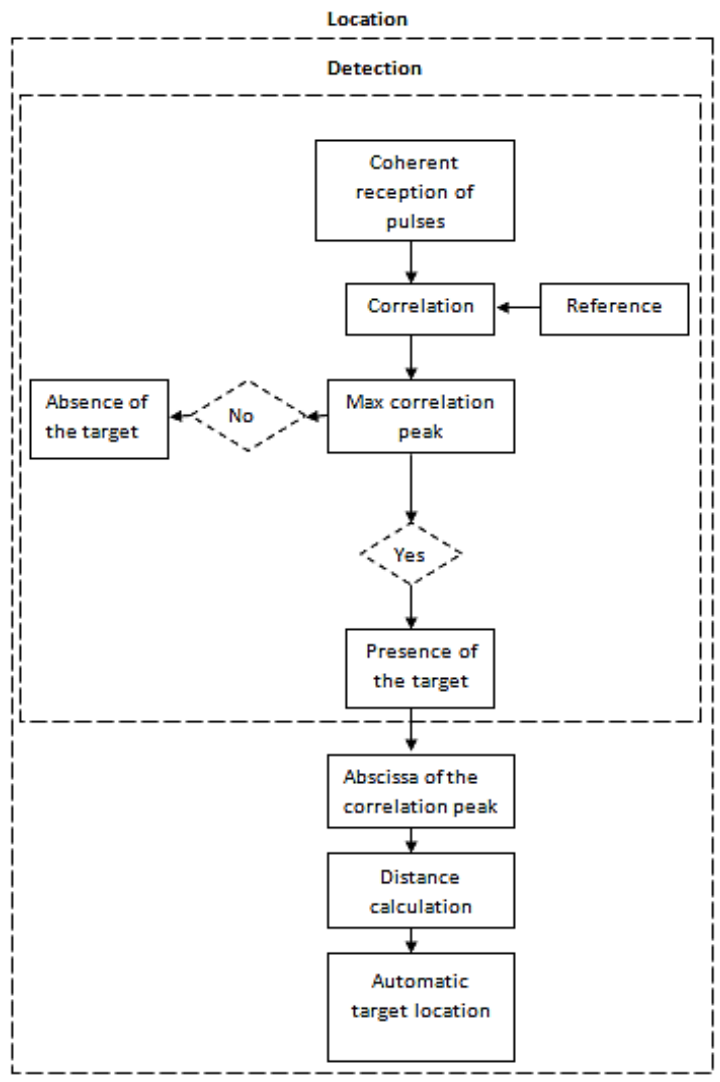

Figure 1. The algorithm for detecting and locating targets 
Correlation is obtained by shifting one of the signals, multiplying the shifted signal by the other signal, and then integrating the resulting signal. For a given waveform and a given target, we obtain a reflected signal specific to them, called a signature, and to know if the target exists or not, we correlate the pulse reflected by the target and the reference signal in order to calculate the distance of the obstacle by determining the position of the correlation peak identified by the threshold detection method. In the following sections, we present the method used and simulation results of different signals used to justify the ability of our algorithm to detect and locate targets in our radar area.

\section{METHOD}

Pulse radar emits a repetitive strain of high-powered signal pulses of brief duration (microwaves) [16]-[20]. Each pulse is followed by a time during which echoes can be received before a new pulse is emitted. The echo processing of the radar pulses provides information about the target, such as the range or distance to the target, determined from the measurement of the propagation time of the electromagnetic wave.

\subsection{UWB detection chain}

The radar system proposed and studied in our research work is a short-range radar based on UWB technology. It is intended for anti-collision applications in road transport. This system is based on the transmission of the unicycle pulse via a Rayleigh vehicle channel [21]; at reception, the correlation technique is applied on the received pulse in order to detect and locate obstacles in the vehicle environment. Figure 2 shows the detection chain proposed for our UWB radar detection.

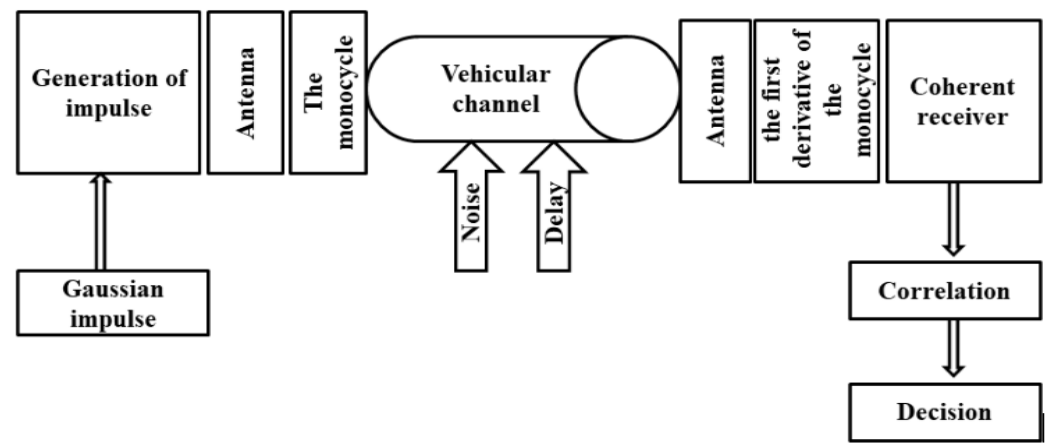

Figure 2. The block diagram of a UWB detection chain

The above transmission chain is based on the generation of a single-cycle pulse with a width of 2 ns at transmission and a frequency of $3 \mathrm{GHz}$, the transmission of this pulse in a vehicular channel that follows a Rayleigh distribution, the reception of the signal by a coherent receiver [22] and finally, the correlation of the received signal with the reference that constitutes the fundamental signal processing step to be able to decide the presence or absence of the obstacle.

\subsection{Signal processing}

On reception, the received signal is processed and analyzed based on the algorithm already presented in the previous section in order to extract the maximum amount of information about the target.

\subsubsection{Received signal}

The radar return signal combines the transmitted signal and the various interference of the propagation channel [23]. This work aims to develop a detection and location system used in very noisy environments such as urban areas. In our case, and to model the real environment, we worked with a multipath vehicle to vehicle channel that follows a Rayleigh distribution reflecting the state of a real space. The following formula models the received signal.

$$
\mathrm{S}(\mathrm{t})=\mathrm{s}(\mathrm{t}) * \mathrm{~h}(\mathrm{t})+\mathrm{n}(\mathrm{t})
$$

With s $(t)$ is the transmitted signal, $h(t)$ represents the impulse response of channel v $2 v$, and $n(t)$ is the Gaussian additive white noise. 


\subsubsection{Target detection and localization}

In the detection step, a decision is made by the detection algorithm regarding the presence or absence of the signal scattered by the target in the radar data. Based on Figure 1, the target can be detected by the UWB radar by correlating the signal backscattered by the latter and picked up by the receiving antenna with the reference in order to measure the degree of similarity between them and to make a decision on the presence of this obstacle in the radar's working area.

The role of radar systems is to detect targets in space where each target is located by its position, direction, and speed [24]. In the case where only the position and direction of the target is determined, the radar system emits through a directional antenna [25], at each instant, a pulse $\mathrm{S}(\mathrm{t})$ only in a narrow angular cone around a determined direction $\theta$. In the presence of a target in this direction, the transmitted signal is reflected and received with a delay time which is proportional to the distance $\mathrm{d}$ between the antenna and the target with:

$$
\begin{aligned}
& \mathrm{R}(\mathrm{t})=\mathrm{S}(\mathrm{t}+\Delta \mathrm{t})+\mathrm{n}(\mathrm{t}) \\
& \Delta \mathrm{t}=2 \mathrm{~d}^{*} \mathrm{c}
\end{aligned}
$$

with $\mathrm{S}(\mathrm{t}+\Delta \mathrm{t})$ is the received signal, $\Delta \mathrm{t}$ is the round-trip time, $\mathrm{d}$ is the distance between the radar and the target, $\mathrm{C}$ is the celerity. After detecting the embedded pulses, the detection scheme identifies correlation peaks and then translates their positions in the target ranges to locate the detected target.

\section{RESULTS AND DISCUSSION}

\subsection{Detection}

The detection of various obstacles by the UWB system, known internationally as UWB [26], is based on the transmission and reception of very short pulses of a few picoseconds or nanoseconds. The advantage of this system is that data transmission rates of the order of a few gigabits per second (Gbps) can be achieved due to the very short pulse duration. The response is very rich in information. The detection phase is used to determine the presence or not of an obstacle in any space. In our case, to be able to decide the presence of the target in the road environment, we carried out a correlation of the received signal with the reference which presents a signal emitted by the transmitting antenna and received by that of reception in the absence of obstacle [27], the signal obtained is analyzed automatically based on a detection algorithm in order to determine the maximum peak of correlation which translates the resemblance between the two signals. Figures 3 and 4 show the transmitted signal translated by a unicycle defined as the first derivative of the Gaussian, the template, the received signal, and the correlation between the latter two signals.

Here the maximum correlation peak reflects the similarity between the two correlated signals, which implies the presence of the target. To test the ability of our algorithm to detect the real signals emitted by our detection radar and reflected by the target, we used an unknown signal [28], [29], and we correlated this signal with the template in order to test the similarity between them. Figures 5 and 6 show an unknown received signal and its correlation with the template. The result obtained after the correlation shows an absence of the correlation peak, which makes it possible to confirm the absence of a copy, delayed in time, of the reference in the signal reflected by the target mentioned above, then the absence of the target in the radar zone.

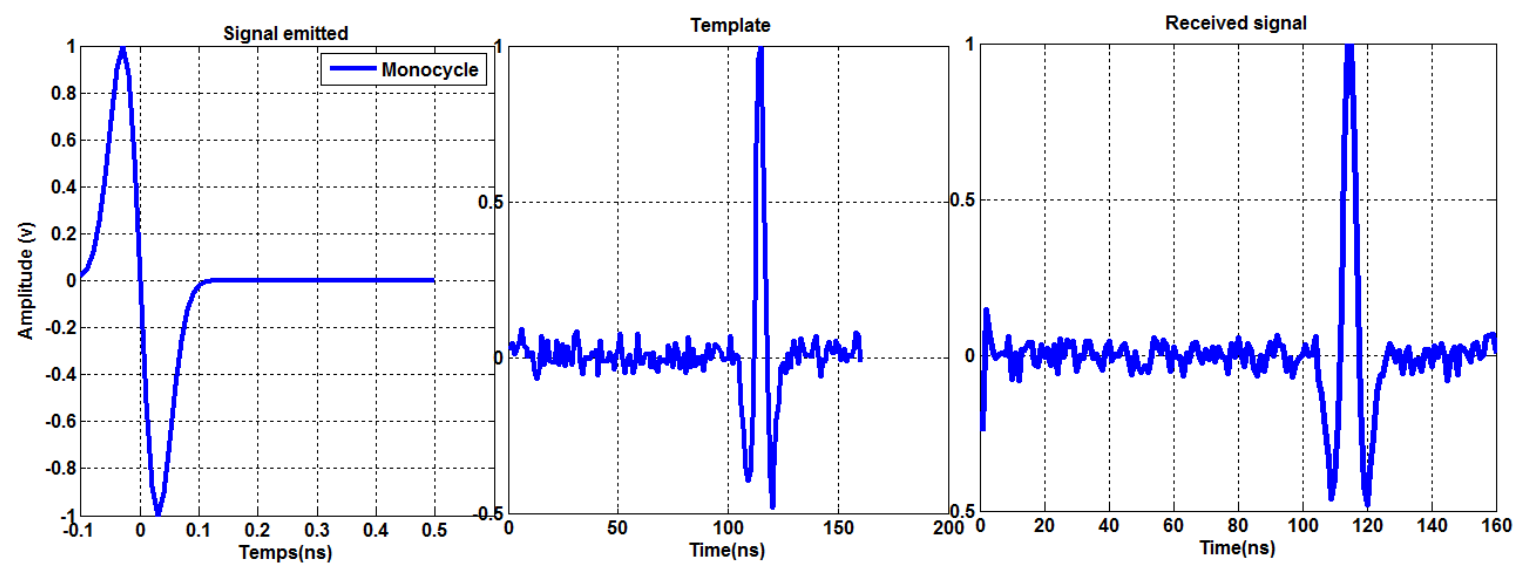

Figure 3. The emitted signal-the reference-received signal 


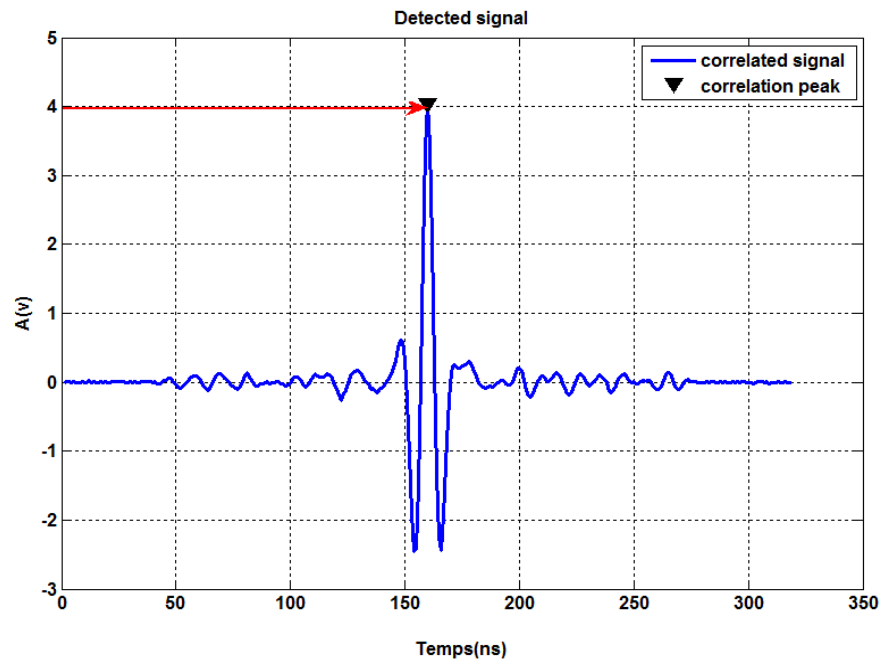

Figure 4. The correlation between the transmitted and received signal

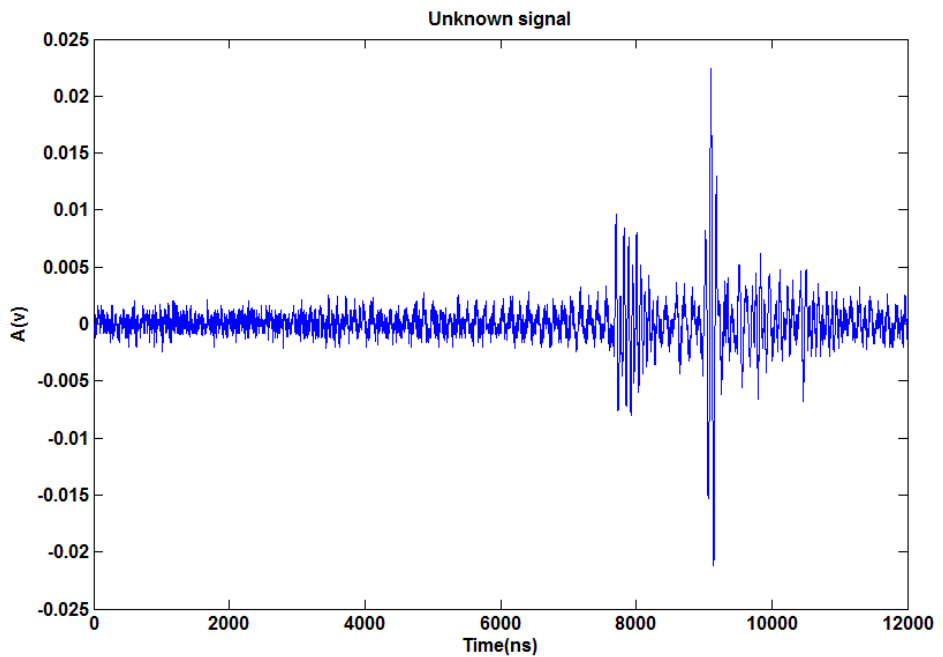

Figure 5. Unknown received signal

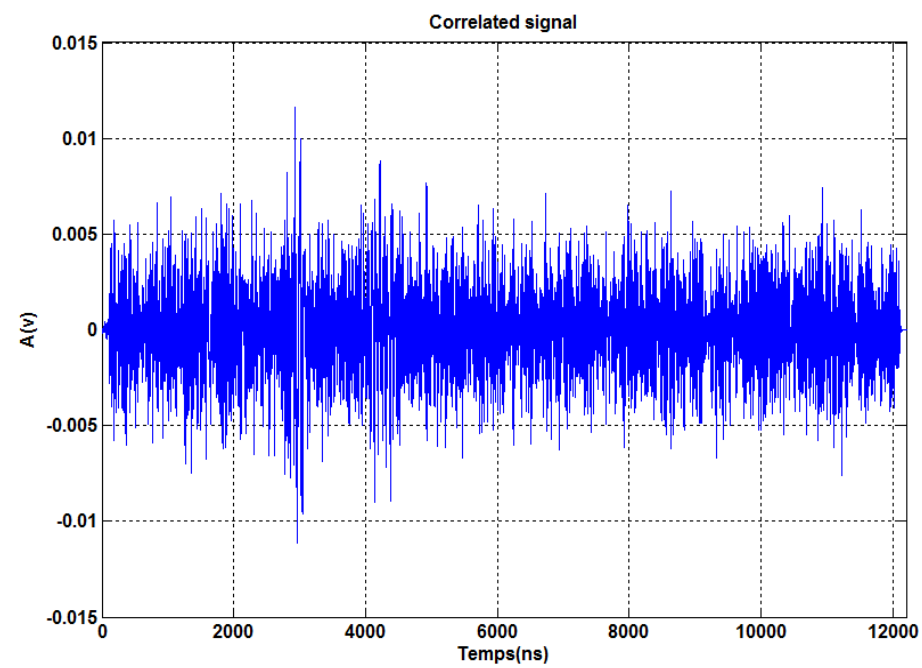

Figure 6. Correlation result of the unknown received signal with the template 


\subsection{Localization}

The objective here is to determine the distance between the radar and the object in the space illuminated by the radar beam. This location is realized from the signal $s(t)$ modeled as the first derivative of the Gaussian pulse (the unicycle) and defined by (3) [11], [27], [30],

$$
S(t)=-2 a\left[\left(\frac{t}{r^{2}}\right) \exp \left[-\left(\frac{t}{r^{2}}\right)\right]\right.
$$

where $a$ is a normalization constant, and $\mathrm{r}$ is the constant used to adjust the width of the pulse. The distance between the obstacle and the radar can be calculated by estimating the flight time by determining the abscissa corresponding to the maximum peak of correlation between the signal reflected by the detected target and the reference. Figure 5 shows the abscissa of the correlation peak used to estimate the time of flight in order to calculate the distance separating the target from the radar. Then, in this case, the distance equal to:

$$
\mathrm{d}=\frac{c \cdot \Delta t}{2}
$$

with $\Delta t$ is the position of the correlation peak that allows determining the location of the target and $\mathrm{C}$ is the celerity. Beyond the detection of the echo, the correlation focuses on the target location, which for the radial direction is obtained by estimating the time of flight. The maximum correlation, identified as the maximum peak, indicates the delay (or time of flight) between transmitted and received pulses. This makes it possible to calculate the distance separating the target from the radar simply and reliably [28]. In our case, the calculated distance equal to $24 \mathrm{~m}$.

\section{CONCLUSION}

In this paper, we presented our UWB radar dedicated to the detection of vulnerable people in urban. Then we discussed the detection and tracking algorithm and, in the end, the simulation results. We have shown that our correlation-based algorithm allows us to detect targets and locate them with better accuracy while determining the maximum correlation peak, which allows us to decide about the presence or not of the targets and then translate their positions which allows us to locate them. Several aspects related to the presented UWB radar remain to be studied and constitute the perspectives of our research work, including the classification and monitoring of obstacles detected.

\section{REFERENCES}

[1] A. Araghi, M. Khalily, P. Xiao, and R. Tafazolli, "Study on the location of mmwave antenna for the autonomous car's detection and ranging sensors," in 2020 14th European Conference on Antennas and Propagation (EuCAP), Mar. 2020, pp. 1-4, doi: 10.23919/EuCAP48036.2020.9135074.

[2] G. Arun Francis, M. Arulselvan, P. Elangkumaran, S. Keerthivarman, and J. Vijaya Kumar, "Object detection using ultrasonic sensor," International Journal of Innovative Technology and Exploring Engineering, vol. 8, no. 6, pp. 207-209, 2019.

[3] A. Alarifi et al., "Ultra wideband indoor positioning technologies: Analysis and recent advances," Sensors (Switzerland), vol. 16, no. 5, May 2016, Art. No. 707, doi: 10.3390/s16050707.

[4] A. H. Majeed and K. H. Sayidmarie, "UWB elliptical patch monopole antenna with dual-band notched characteristics," International Journal of Electrical and Computer Engineering (IJECE), vol. 9, no. 5, pp. 3591-3598, Oct. 2019, doi: 10.11591/ijece.v9i5.pp3591-3598.

[5] J. Ali, R. Yahya, N. Abdullah, and S. Z. Sapuan, "Ultra-wideband monostatic antenna for behind the wall detection," International Journal of Electrical and Computer Engineering (IJECE), vol. 7, no. 6, pp. 2936-2941, Dec. 2017, doi: 10.11591/ijece.v7i6.pp2936-2941.

[6] V. Gharat, E. Colin, G. Baudoin, and D. Richard, "Indoor performance analysis of LF-RFID based positioning system: Comparison with UHF-RFID and UWB," in 2017 International Conference on Indoor Positioning and Indoor Navigation, IPIN 2017, Sep. 2017, vol. 2017-Janua, pp. 1-8, doi: 10.1109/IPIN.2017.8115901.

[7] A. Ren, F. Zhou, A. Rahman, X. Wang, N. Zhao, and X. Yang, "A study of indoor positioning based on UWB base-station configurations," in Proceedings of 2017 IEEE 2nd Advanced Information Technology, Electronic and Automation Control Conference, IAEAC 2017, Mar. 2017, pp. 1939-1943, doi: 10.1109/IAEAC.2017.8054352.

[8] D. Daghouj, S. Mazer, Y. Balboul, A. Menhaj, and L. Sakkila, "Modeling of an obstacle detection chain in a vehicular environment," in 2019 7th Mediterranean Congress of Telecommunications (CMT), Oct. 2019, pp. 1-4, doi: 10.1109/CMT.2019.8931380.

[9] D. Kocur, T. Porteleky, M. Svecova, M. Svingal, and J. Fortes, "A novel signal processing scheme for static person localization using m-sequence UWB radars," IEEE Sensors Journal, vol. 21, no. 18, pp. 20296-20310, Sep. 2021, doi: 10.1109/JSEN.2021.3093658.

[10] P. Dai, Y. Yang, C. Zhang, X. Bao, H. Zhang, and Y. Zhang, "Analysis of target detection based on UWB NLOS ranging modeling," in 2018 Ubiquitous Positioning, Indoor Navigation and Location-Based Services (UPINLBS), Mar. 2018, pp. 1-6, doi: 10.1109/UPINLBS.2018.8559809.

[11] L. Tantiparimongkol and P. Phasukkit, "IR-UWB pulse generation using FPGA scheme for through obstacle human detection," Sensors, vol. 20, no. 13, Jul. 2020, Art. no. 3750, doi: 10.3390/s20133750.

[12] A. Kılıç, İ. Babaoğlu, A. Babalık, and A. Arslan, "Through-wall radar classification of human posture using convolutional neural 
networks," International Journal of Antennas and Propagation, vol. 2019, pp. 1-10, Mar. 2019, doi: 10.1155/2019/7541814.

[13] C. Will, P. Vaishnav, A. Chakraborty, and A. Santra, "Human target detection, tracking, and classification using 24-GHz FMCW radar," IEEE Sensors Journal, vol. 19, no. 17, pp. 7283-7299, Sep. 2019, doi: 10.1109/JSEN.2019.2914365.

[14] O. Rabaste, E. Colin-Koeniguer, D. Poullin, A. Cheraly, J. F. Pétex, and H. K. Phan, "Around-the-corner radar: Detection of a human being in non-line of sight," IET Radar, Sonar and Navigation, vol. 9, no. 6, pp. 660-668, Jul. 2015, doi: 10.1049/ietrsn.2014.0337.

[15] D. Daghouj, M. Fattah, S. Mazer, Y. Balboul, and M. El Bekkali, “UWB waveform for automotive short range radar,” International Journal on Engineering Applications, vol. 8, no. 4, pp. 158-164, Jul. 2020, doi: 10.15866/irea.v8i4.18997.

[16] C. Demonceaux, A. Potelle, and D. Kachi-Akkouche, "Obstacle detection in road scene based on road detection and motion analysis," IFAC Proceedings Volumes (IFAC-PapersOnline), vol. 37, no. 8, pp. 442-447, Jul. 2004, doi: 10.1016/s14746670(17)32016-5.

[17] S. Chang, N. Mitsumoto, and J. W. Burdick, "An algorithm for UWB radar-based human detection," in 2009 IEEE Radar Conference, 2009, pp. 1-6, doi: 10.1109/RADAR.2009.4976999.

[18] A. G. Yarovoy, L. P. Ligthart, J. Matuzas, and B. Levitas, "UWB radar for human being detection," IEEE Aerospace and Electronic Systems Magazine, vol. 21, no. 11, pp. 22-26, Nov. 2006, doi: 10.1109/MAES.2006.284354

[19] K. T. Wong and W. K. Chung, "Pulse-diverse radar/sonar FSK-PSK waveform design to emphasize/de-emphasize designated Doppler-delay sectors," in IEEE National Radar Conference - Proceedings, 2000, pp. 745-749, doi: 10.1109/radar.2000.851928.

[20] D. Cao, T. Li, P. Kang, H. Liu, S. Zhou, and H. Su, "Single-pulse multi-beams operation of phased array radar," in 2016 CIE International Conference on Radar (RADAR), Oct. 2016, pp. 1-4, doi: 10.1109/RADAR.2016.8059597.

[21] M. Hernandez, H.-B. Li, I. Dotlić, and R. M. (NICT), Channel models for TG8. IEEE P802.15 Working Group for Wireless Personal Area Networks (WPANs), 2012.

[22] D. Daghouj, M. Fattah, S. Mazer, Y. Balboul, and M. El Bekkali, "UWB coherent receiver performance in a vehicular channel," International Journal of Advanced Trends in Computer Science and Engineering, vol. 9, no. 2, pp. 1996-2001, Apr. 2020, doi: 10.30534/ijatcse/2020/170922020.

[23] Y. Liu, G. Liao, S. Zhu, Z. Yang, Y. Chen, and X. Zhang, "Performance improvement in a coexistent radar and communications system," in Proceedings of the IEEE Sensor Array and Multichannel Signal Processing Workshop, Jun. 2020, vol. 2020-June, doi: 10.1109/SAM48682.2020.9104303.

[24] W. Yi, T. Zhou, Y. Ai, and R. S. Blum, "Suboptimal low complexity joint multi-target detection and localization for non-coherent MIMO radar with widely separated antennas," IEEE Transactions on Signal Processing, vol. 68, pp. 901-916, 2020, doi: 10.1109/TSP.2020.2968282.

[25] C. Srinivasu, D. Monica Satyavathi, and N. Markandeya Gupta, "Performance evaluation of UWB waveforms in high-resolution radar," in Lecture Notes in Electrical Engineering, vol. 655, Springer Singapore, 2021, pp. 559-565.

[26] C. H. Navitha, K. Sivani, and K. A. Reddy, "Performance evaluation of adaptive continuous wavelet transform based rake receiver for UWB systems," International Journal of Electrical and Computer Engineering (IJECE), vol. 8, no. 5, pp. 3444-3452, Oct. 2018, doi: 10.11591/ijece.v8i5.pp3444-3452.

[27] L. Sakkila, A. Rivenq, F. Boukour, C. Tatkeu, Y. El Hillali, and J. M. Rouvaen, "Collision avoidance radar system using UWB waveforms signature for road applications," in 2009 9th International Conference on Intelligent Transport Systems Telecommunications, ITST 2009, Oct. 2009, pp. 223-226, doi: 10.1109/ITST.2009.5399353.

[28] M. Abdellaoui, D. Daghouj, M. Fattah, Y. Balboul, S. Mazer, and M. El Bekkali, "Artificial intelligence approach for target classification: A state of the art," Advances in Science, Technology and Engineering Systems, vol. 5, no. 4, pp. 445-456, Aug. 2020, doi: $10.25046 / \mathrm{AJ} 050453$.

[29] A. Maroua and F. Mohammed, "Characterization of ultra wide band indoor propagation," in 2019 7th Mediterranean Congress of Telecommunications (CMT), Oct. 2019, pp. 1-4, doi: 10.1109/CMT.2019.8931367.

[30] M. Fattah et al., "Multi band OFDM alliance power line communication system," Procedia Computer Science, vol. 151, pp. 1034-1039, 2019, doi: 10.1016/j.procs.2019.04.146.

\section{BIOGRAPHIES OF AUTHORS}

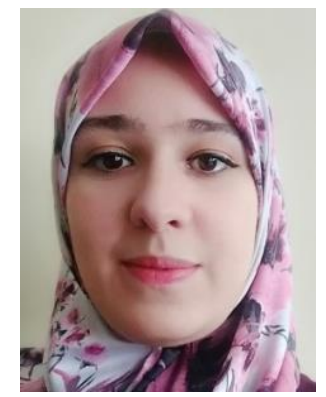

Dounia Daghouj (iD 8I SC P was born in Taza in 1992. She obtained her diploma of state engineer in network and telecommunication from the National School of Applied Sciences in 2016; currently he is a PhD student in Artificial Intelligence, Data Sciences and Emerging Systems Laboratory at the University of Sidi Mohamed Ben Abdellah Fez. She could be contacted via email: dounia.daghouj@usmba.ac.ma.

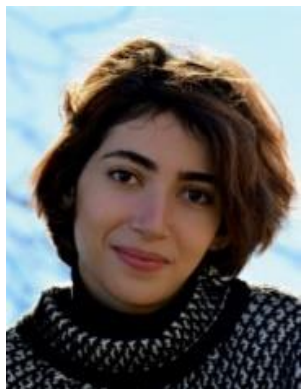

Maroua abdellaoui (D) 81 SC $\mathrm{P}$ obtained her diploma of state engineer in network and telecommunication from the National School of Applied Sciences in 2017; currently he is a $\mathrm{PhD}$ student in Artificial Intelligence, Data Sciences and Emerging Systems Laboratory at the University of Sidi Mohamed Ben Abdellah. She could be contacted via email: abdellaoui.marwa@gmail.com. 


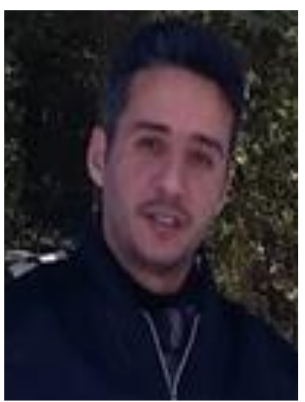

Mohammed Fattah (D) SC P received his Ph.D. in Telecommunications and CEM at the University of Sidi Mohamed Ben Abdellah (USMBA) Fez, Morocco, 2011. He is a professor in the Electrical Engineering Department of the High school of technology at the Moulay Ismail University (UMI), Meknes, Morocco and he is a responsible of the research team 'Intelligent Systems, Networks and Telecommunications', IMAGE laboratory, UMI. He could be contacted via email: fattahm@gmail.com.

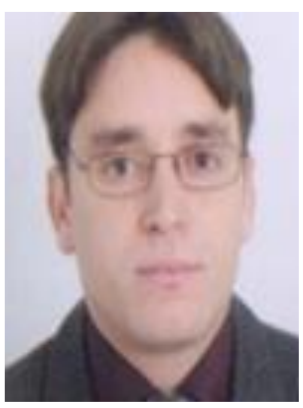

Said Mazer (iD PII SC P was born in 1978. He has his PhD in electronics and signal processing at the University of Marne-La-Vallée. Currently a professor at the National School of Applied Sciences in Fez, Morocco, he is a member of the Artificial Intelligence, Information Sciences and Emerging Systems Laboratory o the University of Sid Mohamed Ben Abdellah Fez. He could be contacted via email: mazersaid@gmail.com.

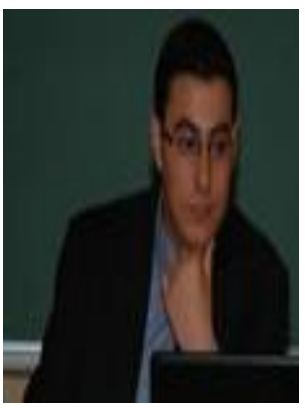

Younes Balboul (iD) IS SC P received his Ph.D. in Telecommunications at the University of Sidi Mohamed Ben Abdellah (USMBA) Fez, Morocco, 2016. Currently professor at the National School of Applied Sciences of Fez, Morocco and member of Artificial Intelligence, Data Sciences and Emerging Systems Laboratory at the University of Sidi Mohamed Ben Abdellah Fez. He could be contacted via email: balboulyounes@gmail.com.

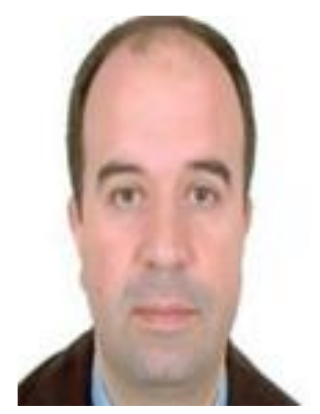

Moulhime El Bekkali (D) 8D SC P received his PhD in1991 at the USTL, University of Lille 1, France, where he worked on X-band printed antennas and their applications to microwave radars. He is a professor in the Department of Electrical Engineering at the Superior School of Technology, Fez (ESTF). He is a member of the Artificial Intelligence, Data Science and Emerging Systems Laboratory. Since 2009; Prof. El Bekkali has been Vice- President of the Sidi Mohamed Ben Abdellah University (USMBA) in Fez, Morocco. He could be contacted via email: Moulhime.elbekkali@usmba.ac.ma. 